\title{
Implementing ACOVE Quality Indicators as an Intervention Checklist to Improve Care for Hospitalized Older Adults
}

\author{
Liron Sinvani, MD¹, Andrzej Kozikowski, $\mathrm{PhD}^{2 \star}$, Christopher Smilios, MPH², Vidhi Patel, MS², Guang Qiu, MD², \\ Meredith Akerman, $\mathrm{MS}^{3}$, Martin Lesser, $\mathrm{PhD}^{3}$, David Rosenberg, MD, MPH${ }^{1}$, Gisele Wolf-Klein, MD4, Renee Pekmezaris, PhD²
}

${ }^{1}$ Divisions of Hospital Medicine, Northwell Health, Manhasset, New York; ${ }^{2}$ Department of Medicine, Northwell Health, Manhasset, New York; ${ }^{3}$ The Feinstein Institute for Medical Research, Manhasset, New York; ${ }^{4}$ Geriatric \& Palliative Medicine, Northwell Health, Manhasset, New York.

BACKGROUND: Medicare patients account for approximately $50 \%$ of hospital days. Hospitalization in older adults often results in poor outcomes.

OBJECTIVE: To test the feasibility and impact of using Assessing Care of Vulnerable Elders (ACOVE) quality indicators (QIs) as a therapeutic intervention to improve care of hospitalized older adults.

DESIGN: Post-test only prospective intervention with a nonequivalent retrospective control group.

SETTING: Large tertiary hospital in the greater New York Metropolitan area.

PATIENTS: Hospitalized patients, 75 years and over, admitted to medical units.

INTERVENTION: A checklist, comprised of four ACOVE QIs, administered during daily interdisciplinary rounds: venous thrombosis prophylaxis (VTE) (QI 1), indwelling bladder catheters (Q। 2), mobilization (Q। 3), and delirium evaluation (Q। 4).

MEASUREMENTS: Variables were extracted from electron- ic medical records with QI compliance as primary outcome, and length of stay (LOS), discharge disposition, and readmissions as secondary outcomes. Generalized linear mixed models for binary clustered data were used to estimate compliance rates for each group (intervention group or control group) in the postintervention period, along with their corresponding 95\% confidence intervals.

RESULTS: Of the 2,396 patients, 530 were on an intervention unit. In those patients not already compliant with VTE, compliance rate was $57 \%$ in intervention vs $39 \%$ in control $(P<$ .0056). For indwelling catheters, mobilization, and delirium evaluation, overall compliance was significantly higher in the intervention group $72.2 \%$ vs $54.4 \%(P=.1061), 62.9 \%$ vs $48.2 \%$ $(P<.0001)$, and $27.9 \%$ vs $21.7 \%(P=.0027)$, respectively.

CONCLUSION: The study demonstrates the feasibility and effectiveness of integrating ACOVE Qls to improve the quality of care in hospitalized older adults. Journal of Hospital Medicine 2017;12:517-522. (C) 2017 Society of Hospital Medicine
In 2014, the United States spent $\$ 3$ trillion on healthcare; hospitalization consumed $32 \%$ of these expenditures. ${ }^{1}$ Today, Medicare patients account for over $50 \%$ of hospital days and over $30 \%$ of all hospital discharges in the United States. ${ }^{2}$ Despite this staggering financial burden, hospitalization of older adults often results in poor patient outcomes. ${ }^{3-6}$ The exponential growth of the hospitalist movement, from 350 hospitalists nationwide in 1995 to over 44,000 in 2014, has become the key strategy for providing care to hospitalized geriatric patients. ${ }^{7-10}$ Most of these hospitalists have not received geriatric training. ${ }^{11-15}$

There is growing evidence that a geriatric approach, emphasizing multidisciplinary management of the complex needs of older patients, leads to improved outcomes. Geriatric Evaluation and Management Units (GEMUs), such as Acute Care for Elderly (ACE) models, have demonstrated

\footnotetext{
*Address for correspondence and reprint requests: Andrzej Kozikowski, Northwell Health, 175 Community Drive, Second Floor, Great Neck, NY 11021; Telephone: (516) 465-3211; Fax: (516) 465-2699; E-mail: akozikowski@ northwell.edu
}

Received: August 25, 2016; Revised: November 30, 2016; Accepted: December 9, 2016

2017 Society of Hospital Medicine DOI 10.12788/jhm.2765 significant decreases in functional decline, institutionalization, and death in randomized controlled trials. ${ }^{16,17}$ Multidisciplinary, nonunit based efforts, such as the mobile acute care of elderly (MACE), proactive consultation models (Sennour/Counsell), and the Hospital Elder Life Program (HELP), have demonstrated success in preventing adverse events and decreasing length of stay (LOS). ${ }^{17-20}$

However, these models have not been systematically implemented due to challenges in generalizability and replicability in diverse settings. To address this concern, an alternative approach must be developed to widely "generalize" geriatric expertise throughout hospitals, regardless of their location, size, and resources. This initiative will require systematic integration of evidence-based decision support tools for the standardization of clinical management in hospitalized older adults. ${ }^{21}$

The 1998 Assessing Care of Vulnerable Elders (ACOVE) project developed a standardized tool to measure and evaluate the quality of care by using a comprehensive set of quality indicators (QIs) to improve the care of "vulnerable elders" (VEs) at a high risk for functional and cognitive decline and death. ${ }^{22-24}$ The latest systematic review concludes that, although many studies have used ACOVE as an assessment tool of quality, there has been a dearth of studies investigating 
the ACOVE QIs as an intervention to improve patient care. ${ }^{25}$

Our study investigated the role of ACOVE as an intervention by using the QIs as a standardized checklist in the acute care setting. We selected the 4 most commonly encountered QIs in the hospital setting, namely venous thrombosis prophylaxis (VTE), indwelling bladder catheter, mobilization, and delirium evaluation, in order to test the feasibility and impact of systematically implementing these ACOVE QIs as a therapeutic intervention for all hospitalized older adults.

\section{METHODS}

This study (IRB \#13-644B) was conducted using a prospective intervention with a nonequivalent control group design comprised of retrospective chart data from May 1, 2014, to June 30, 2015. Process and outcome variables were extracted from electronic medical records ([EMR], Sunrise Clinical Manager [SCM]) of 2,396 patients, with 530 patients in the intervention unit and 1,866 on the control units, at a large academic tertiary center operating in the greater New York metropolitan area. Our study investigated the role of ACOVE as an intervention to improve patient care by using selected QIs as a standardized checklist tool in the acute care setting. Of the original 30 hospital-specific QIs, our study focused on the care of older adults admitted to the medicine service. ${ }^{26}$ We selected commonly encountered QIs, with the objective of testing the feasibility and impact of implementing the ACOVE QIs as an intervention to improve care of hospitalized older adults. This intervention consisted of applying the checklist tool, constructed with 4 selected ACOVE QIs and administered daily during interdisciplinary rounds, namely: 2 general "medical" indicators, VTE prophylaxis and indwelling bladder catheters, and 2 "geriatric"-focused indicators, mobilization and delirium evaluation.

Subject matter experts (hospitalists, geriatricians, researchers, administrators, and nurses) reviewed the ACOVE QIs and agreed upon the adaptation of the QIs from a quality measure assessment into a feasible and acceptable intervention checklist tool (Table 1). The checklist was reviewed during daily interdisciplinary rounds for all patients 75 years and older. While ACOVE defined vulnerable elders by using the Vulnerable Elder Screen (VES), we wanted to apply this intervention more broadly to all hospitalized older adults who are most at risk for poor outcomes. ${ }^{27}$ Patients admitted to the intensive care unit, inpatient psychiatry, inpatient leukemia/lymphoma, and surgical services were excluded.

Daily interdisciplinary rounds are held on every one of the five 40-bed medical units; they last approximately 1 hour, and consist of a lead hospitalist, nurse manager, nurse practitioners, case managers, and the nursing staff. During interdisciplinary rounds, nurses present the case to the team members who then discuss the care plan. These 5 medical units did not differ in terms of patient characteristics or staffing patterns; the intervention unit was chosen simply for logistical reasons, in that the principal investigator (PI) had been assigned to this unit prior to study start-up.

Prior to the intervention, LS held an education session for staff on the intervention unit staff (who participated on inter- disciplinary rounds) to explain the concept of the ACOVE QI initiative and describe the four QIs selected for the study. Three subsequent educational sessions were held during the first week of the intervention, with new incoming staff receiving a brief individual educational session. The staff demonstrated significant knowledge improvement after session completion (pre/post mean score $70.6 \%$ vs $90.0 \% ; P<.0001$ ).

The Clinical Information System for the Health System EMR, The Eclipsys SCM, has alerts with different levels of severity from "soft" (user must acknowledge a recommendation) to "hard" (requires an action in order to proceed).

To measure compliance of the quality indicators, we collected the following variables:

\section{QI 1: VTE prophylaxis}

Through SCM, we collected type of VTE prophylaxis ordered (pharmacologic and/or mechanical) as well as start and stop dates for all agents. International normalized ratio levels were checked for patients receiving warfarin. Days of compliance were calculated.

\section{QI 2: Indwelling Bladder Catheters}

SCM data were collected on catheter entry and discontinuation dates, the presence of an indication, and order renewal for bladder catheter at least every 3 days.

\section{QI 3: Mobilization}

Ambulation status prior to admission was extracted from nursing documentation completed on admission to the medical ward. Patients documented as bedfast were categorized as nonambulatory prior to admission. Nursing documentation of activity level and amount of feet ambulated per nursing shift were collected. In addition, hospital day of physical therapy (PT) order and hospital days with PT performed were charted. Compliance with QI 3 in patients documented as ambulatory prior to hospital admission was recorded as present if there was a PT order within 48 hours of admission.

\section{QI 4: Delirium Evaluation}

During daily rounds, the hospitalist (PI) questioned nurses about delirium evaluation, using the first feature of the Confusion Assessment Method (CAM) as well as the "single question in delirium," namely, "Is there evidence of an acute change in mental status from the patient's baseline?" and "Do you think [name of patient] has been more confused lately?"28,29 Because EMR does not contain a specified field for delirium screening and documentation, and patients are not routinely included in rounds, documentation with QI 4 was recorded using the "key words" method as described in the work by Puelle et al. ${ }^{30}$ To extract SCM key words, nursing documentation of the "cognitive/ perceptual/neurological exam" section of the EMR on admission and on all subsequent documentation (once per shift) was retrieved to identify acute changes in mental status (eg, "altered mental status, delirium/delirious, alert and oriented X 3, confused/confusion, disoriented, lethargy/lethargic"). ${ }^{30}$ In addition, nurses were asked to activate an SCM parameter, "Acute Con- 
TABLE 1. Integration of Selected ACOVE QIs Into a Checklist-Based Tool for Decision Support

\begin{tabular}{|c|c|c|c|}
\hline Patient Condition & ACOVE Rationale & Checklist/Decision Support Tool & QI Implementation \\
\hline $\begin{array}{l}\text { Venous Thrombosis } \\
\text { Prophylaxis }\end{array}$ & $\begin{array}{l}\text { IF a hospitalized VE is at high risk for venous thrombosis, } \\
\text { THEN s/he should receive DVT prophylaxis, BECAUSE } \\
\text { large randomized placebo-controlled trials of VTE } \\
\text { prophylaxis in hospitalized medical patients demonstrate } \\
\text { a } 40 \%-63 \% \text { reduction in VTE. }{ }^{31}\end{array}$ & $\begin{array}{l}\text { 1. Identification of high-risk vulnerable elderly hospital- } \\
\text { ized patients for venous thrombosis, and } \\
\text { 2. Ordering venous thrombosis prophylaxis (pharmaco- } \\
\text { logical/mechanical) if indicated }\end{array}$ & $\begin{array}{l}\text { The presence and appropriateness of DVT prophylaxis is } \\
\text { discussed, with the goal of determining whether the patient } \\
\text { is receiving appropriate DVT prophylaxis, and whether any } \\
\text { modifications should occur in accordance with the QI. }\end{array}$ \\
\hline
\end{tabular}

Indwelling Bladder IF a hospitalized VE has an indwelling bladder catheter Catheter placed. THEN the indication or continued need for the catheter should be documented at least every 3 days until its removal, BECAUSE indwelling bladder catheters are often placed without an appropriate indication, and many physicians are unaware that their hospitalized patients are catheterized. ${ }^{3}$
1. Documentation of catheter need at least every 3 days until removal

2. Documentation of catheter indication
Specifically, the presence, indication, and possible remova of the indwelling bladder catheter was discussed for each patient who meets the inclusion criteria, with the goal of determining whether indwelling bladder catheter removal is indicated.
Mobilization

IF a VE who is ambulatory as an outpatient is hospitalized for longer than 48 hours and is not receiving intensive or palliative care, THEN there should be a plan to increase mobility within 48 hours of admission, BECAUSE early ambulation can reduce length of stay for hospitalized VES by approximately one day. ${ }^{3}$

\author{
1. Determining ambulatory status of the patient prior to \\ admission based on nursing admission documentation \\ 2. If a patient was ambulatory prior to admission, the \\ patient should be ambulated at least daily \\ 3. The nursing staff should document daily the approxi- \\ mate number of feet ambulated \\ 4. The clinical should consider a physical therapy evalua- \\ tion to increase mobility within 48 hours of admission
}

To ensure that patients ambulatory on admission remain mobile, the status of a mobility plan was evaluated by the interdisciplinary team, encouraging nursing staff to get the patient out of bed, and encouraging the nurse to document approximate amount of feet the patient ambulated and triggering the order of a physical therapy consult, when appropriate, within 48 hours of admission.

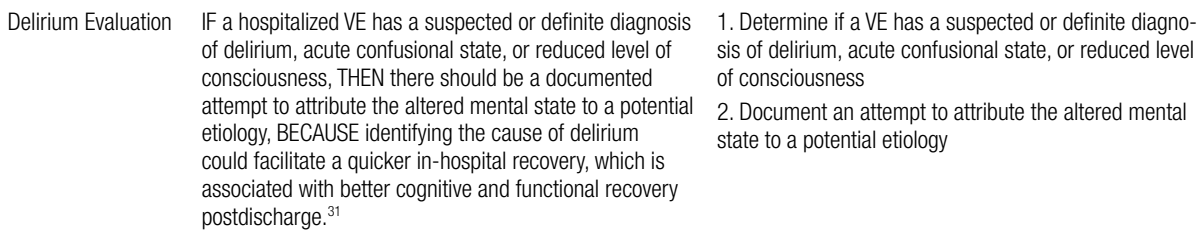
of delirium, acute confusional state, or reduced level of consciousness, THEN there should be a documented attempt to attribute the altered mental state to a potential etiology, BECAUSE identifying the cause of delirium could facilitate a quicker in-hospital recovery, which is associated with better cognitive and functional recovery postdischarge. ${ }^{31}$

1. Determine if a VE has a suspected or definite diagnosis of delirium, acute confusional state, or reduced level of consciousness

2. Document an attempt to attribute the altered mental state to a potential etiology

Nurses are asked on a daily basis if there is any evidence of acute confusion or a change from baseline. Is there evidence of acute confusion? ${ }^{40}$ If the patient had acute confusion the nurse is instructed to open a parameter for acute confusion and document the potential cause/s.

NOTE: Abbreviations: ACOVE, Assessing Care of Vulnerable Elders; DVT, deep vein thrombosis; Ql, quality indicator; VE, vulnerable elder; VTE, venous thromboembolism. Source: Arora VM, McGory ML, Fung CH. Quality indicators for hospitalization and surgery in vulnerable elders. J Am Geriatr Soc. 2007;55(S2):S347-S358.

fusion" SCM parameter, in the nursing documentation section, which includes potential risk factors for confusion.

In addition to QI compliance, we collected LOS, discharge disposition, and 30-day readmission data.

Generalized linear mixed models (GLMM) for binary clustered (ie, hierarchical) data were used to estimate compliance rates (ie, nurse adherence) for each group (intervention group or control group) in the postintervention period, along with their corresponding 95\% confidence intervals. GLMM was used to account for the hierarchical structure of the data: nursing units within a hospital. In order to calculate the Charlson Comorbidity Index, we extracted past medical history from the EMR. ${ }^{31}$

Subjects $(N=2,396)$ were included in the comparison of the intervention group vs control group for each of the following 4 ACOVE QI compliance measures: DVT, mobilization, bladder catheter, and delirium.

\section{RESULTS}

Of the 2,396 patient admissions, 530 were in the intervention unit and 1,866 were in the control unit. In the inter- vention group, the average age was 84.65 years, $75.58 \%$ were white and $47.21 \%$ were married. There was no difference in patient demographics between groups (Table 2).

\section{QI 1: VTE Prophylaxis}

Compliance with VTE prophylaxis was met in $78.3 \%$ of the intervention subjects and $76.5 \%$ of the controls $(P<.4371)$ (Table 3). Of note, the rate of VTE prophylaxis was $57 \%$ in the intervention vs $39 \%$ in the control group $(P<.0056)$, in the 554 patients for whom compliance was not met. Mechanical prophylaxis was used in $35.6 \%$ of intervention subjects vs 30.6 in the control $(P=.048)$. Patients who received no form of prophylaxis were $0.5 \%$ in the intervention and $3 \%$ in the control $(P=.027)$.

\section{QI 2: Indwelling Bladder Catheters}

Out of 2,396 subjects, 406 had an indwelling bladder catheter $(16.9 \%)$. Compliance with the catheter was met in $72.2 \%$ of the intervention group vs $54.4 \%$ in the control group $(P=$ .1061). An indication for indwelling bladder catheters was documented in $100 \%$ of the subjects. The average number of 
TABLE 2. Patient Characteristics

\begin{tabular}{|c|c|c|c|}
\hline Demographics & $\begin{array}{c}\text { Control } \\
(\mathrm{N}=1505)\end{array}$ & $\begin{array}{l}\text { Intervention } \\
(\mathrm{N}=430)\end{array}$ & $P$ value \\
\hline Age, mean (SD) & $84.69(6.21)$ & $84.65(6.04)$ & .8890 \\
\hline BMI, mean (SD) & $26.11(5.86)$ & $25.40(5.44)$ & .0607 \\
\hline Charlson Comorbidity Score, mean (SD) & $6.44(1.83)$ & $6.35(1.74)$ & .4434 \\
\hline Gender, n (\%) & & & .4415 \\
\hline Male & $592(39.34)$ & $178(41.40)$ & \\
\hline Female & $913(60.66)$ & $252(58.60)$ & \\
\hline Race, n (\%) & & & .9351 \\
\hline White & $1125(74.75)$ & $325(75.58)$ & \\
\hline Black & $135(8.97)$ & $37(8.60)$ & \\
\hline Asian & $45(2.99)$ & $16(3.72)$ & \\
\hline Native American/Pacific Islander & $2(0.13)$ & $1(0.23)$ & \\
\hline Other & $159(10.56)$ & $41(9.53)$ & \\
\hline Not Specified & $39(2.59)$ & $10(2.33)$ & \\
\hline Marital Status, n (\%) & & & .6069 \\
\hline Married & $664(44.12)$ & $203(47.21)$ & \\
\hline Single & 159 (10.56) & $53(12.33)$ & \\
\hline Separated & $5(0.33)$ & $1(0.23)$ & \\
\hline Divorced & $51(3.39)$ & 13 (3.02) & \\
\hline Widowed & $573(38.07)$ & 145 (33.72) & \\
\hline Not Specified & 53 (3.52) & $15(3.49)$ & \\
\hline
\end{tabular}

NOTE: Abbreviations: BMI, body mass index; SD, standard deviation.

catheter days was 5.16 in the intervention vs 5.88 in the control $(P<.2284)$. There was statistical significance in catheter compliance in the longer stay ( $>15$ days) subjects, decreasing to $23.32 \%$ in the control group while staying constant in the intervention group $71.5 \%(P=.0006)$.

\section{QI 3: Mobilization}

Of the 2,396 patients, 1,991 (83.1\%) were reported as ambulatory prior to admission. In the intervention vs control group, 74 (14\%) vs 297 (15.7\%), respectively, were nonambulatory. Overall compliance with Q3 was $62.9 \%$ in the intervention vs $48.2 \%$ in the control $(P<.0001)$. More specifically, the average time to PT order in the intervention group was 1.83 days vs 2.22 days in the control group $(P<$ $.0051)$ and the time to PT evaluation was 2.14 days vs 2.42 days, respectively $(P<.0108)$. In the intervention group, 84 patients $(15.8 \%)$ did not have a PT consult vs $511(27 \%)$ in the control group $(P<.0001)$. The average times per subject in which the nurses documented the approximate number of feet ambulated was 6.48 in the intervention group vs 0.11 in the control group.

\section{QI 4: Delirium Evaluation}

In terms of nursing documentation indicating the presence of an acute confusional state, the intervention group had 148 out of 530 nursing notes $(27.9 \%)$ vs 405 out of 1,866 in the control group $(21.7 \% ; P=.0027)$. However, utilization of the "acute confusion" parameter with documentation of a risk factor did not differ between the groups (5.8\% in the intervention group vs $5.6 \%$ in the control group, $P<.94$ ).

\section{LOS, Discharge Disposition, and 30-Day Readmissions}

LOS did not differ between intervention and control groups (6.37 days vs 6.27 days, respectively), with a median of 5 days $(P=.877)$. Discharge disposition in the 2 groups included the following: home/home with services $(71.32 \%$ vs $68.7 \%$ ), skilled nursing facility/assisted living/long-term care (24.34 versus 25.83), inpatient hospice/home hospice (2.64 vs 2.25$)$, and expired (1.13 vs $1.77 ; P<.3282)$. In addition, 30 -day readmissions did not differ $(21 \%$ vs $20 \%$, respectively, $P=.41$ ).

\section{DISCUSSION}

Our goal was to explore an evidence-based, standardized approach to improve the care of hospitalized older adults. This approach leverages existing automated EMR alert functions with an additional level of decision support for VEs, integrated into daily multidisciplinary rounds. The use of a daily checklist-based tool offers a cost-effective and practical pathway to distribute the burden of compliance responsibility amongst team members.

As we anticipated and similar to study findings in hospitalized medicine, geriatric trauma, and primary care, compliance with general care QIs was better than geriatric-focused QIs. ${ }^{27,32}$ Wenger et $\mathrm{al}^{33}$ demonstrated significant improvements with screening for falls and incontinence; however, screening for cognitive impairment did not improve in the outpatient setting by imbedding ACOVE QIs into routine physician practice.

Increased compliance with VTE prophylaxis and indwelling bladder catheters may be explained by national financial incentives for widespread implementation of EMR alert systems. Conversely, mobilization, delirium assessment, and management in hospitalized older adults don't benefit from similar incentives.

\section{VTE Prophylaxis}

The American College of Chest Physicians (ACCP) supports the use of VTE prophylaxis, especially in hospitalized older adults with decreased mobility. ${ }^{34}$ While greater adoption of EMR has already increased adherence, our intervention resulted in an even higher rate of compliance with the use of pharmacologic VTE prophylaxis. ${ }^{35}$ In the future, validated scores for risk of thrombosis and bleeding may be integrated into our QI-based checklist.

\section{Indwelling Bladder Catheters}

The potential harms of catheters have been described for over 50 years, yet remain frequently used. ${ }^{36,37}$ Previous studies have shown success in decreasing catheter days with computer-based and multidisciplinary protocols. ${ }^{36-39}$

Our health system's EMR has built-in "soft" and "hard" alerts for indwelling bladder catheters, so we did not expect intervention-associated changes in compliance. 


\section{Mobilization}

Hospitalization in older adults frequently results in functional decline. ${ }^{4,5,40}$ In response, the mobilization QI recommends an ambulation plan within 48 hours for those patients who were ambulatory prior to admission; it does not specifically define the components of the plan. ${ }^{26}$ There are several multicomponent interventions that have demonstrated improvement in functional decline, yet they require skilled providers. ${ }^{41,42}$ Our intervention implemented specific ambulation plan components: daily ambulation and documentation reminders and early PT evaluation.

While functional status measures have existed for decades, most are primarily geared to assess community-residing individuals and not designed to measure changes in function during hospitalization. ${ }^{43,44}$ Furthermore, performance-based hospital measures are difficult to integrate into the daily nursing workflow as they are time consuming. ${ }^{45,46}$ In practice, nurses routinely use free text to document functional status in the hospital setting, rendering comparative analysis problematic. Yet, we demonstrated that nurses were more engaged in reporting mobilization (increased documentation of ambulation distance and a decrease in time to PT). Future research should focus on the development of a standardized tool, integrated into the EMR, to accurately measure function in the acute care setting.

\section{Delirium Evaluation}

Delirium evaluation remains one of the most difficult clinical challenges for healthcare providers in hospitalized individuals, and our study reiterated these concerns. Previous research has consistently demonstrated that the diagnosis of delirium is missed by up to $75 \%$ of clinicians. ${ }^{47,48}$ Indeed, our study, which exclusively examined nursing documentation of the delirium evaluation QI, found that both groups showed strikingly low compliance rates. This may have been due to the fact that we only evaluated nursing documentation of suspected or definite diagnosis of delirium and a documented attempt to attribute the altered mental state to a potential etiology. ${ }^{31}$ By utilizing the concept of "key words," as developed by Puelle et al. ${ }^{30}$, we were able to demonstrate a statistically significant improvement in nursing delirium documentation in the intervention group. This result should be interpreted with caution, as this approach is not validated. Furthermore, our operational definition of delirium compliance (ie, nurse documentation of delirium, requiring the launching of a separate parameter) may have been simply too cumbersome to readily integrate into the daily workflow. Future research should study the efficacy of a sensitive EMR-integrated screening tool that facilitates recognition, by all team members, of acute changes in cognition.

Although a number of QI improved for the intervention group, acute care utilization measures such as LOS, discharge disposition, and 30-day readmissions did not differ between groups. It may well be that improving quality for this very frail, vulnerable population may simply not result in decreased utilization. Our ability to further decrease LOS
TABLE 3. Quality Indicator Outcomes/Measurements

\begin{tabular}{llccc}
\hline QI & QI Compliance & Control & Intervention & $P$ value \\
\hline Q11 & Venous Thrombosis Prophylaxis (\%) & 76.5 & 78.3 & $<.4371$ \\
\hline & Mechanical Prophylaxis & 30.56 & 35.58 & .0486 \\
\hline Q12 & Q11: Indwelling Bladder Catheters (\%) & 54.43 & 72.15 & .1061 \\
\hline & Average number of catheter days & 5.88 & 5.16 & $<.2284$ \\
\hline Q13 & Mobilization (\%) & 48.2 & 62.9 & $<.0001$ \\
\hline Ambulatory PTA (\%) & 84.3 & 86 & \\
\hline & Average time to PT order (days) & 2.22 & 1.83 & $<.0051$ \\
\hline Time to PT evaluation (days) & 2.42 & 2.14 & $<.0108$ \\
\hline Q14 & Delirium Documentation (\%) & 21.7 & 27.9 & .0027 \\
\hline & Compliance of delirium documentation and RF & 5.8 & 5.6 & $<.94$ \\
\hline
\end{tabular}

NOTE: Abbreviations: PT, physical therapy; PTA, prior to admission; QI, quality indicator; RF, risk factor.

and readmission rates may be limited due to restriction of range in this complex patient population (eg, median LOS value of 5 days).

\section{Limitations}

Although our study had a large sample size, data were only collected from a single-center and thus require further exploration in different settings to ensure generalizability. In addition, QI observance was based on the medical record, which was problematic for some indicators, notably delirium identification. While prior literature highlights the difficulty in identifying delirium, especially during clinical practice without specialized training, our compliance was strikingly low. ${ }^{47}$ While validated measures such as CAM may have been included as part of the assessment, there is currently no EMR documentation of such measures and therefore, these data could not be obtained.

\section{CONCLUSION}

In summary, our study demonstrates the successful integration of the established ACOVE QIs as an intervention, rather than as an assessment method, for improving care of hospitalized older patients. By utilizing a checklist-based tool at the bedside allows the multidisciplinary team to implement evidence-based practices with the ultimate goal of standardizing care, not only for VEs, but potentially for other highrisk populations with multimorbidity. ${ }^{49}$ This innovative approach provides a much-needed direction to healthcare providers in the ever increasing stressful conditions of today's acute care environment and for the ultimate benefit and safety of our older patients.

Disclosure: The authors declare no conflicts of interest. This study was supported by New York State Empire Clinical Research Investigators Program (ECRIP). The sponsor had 
no role in the conception, study design, data collection, data analysis, interpretation of data, manuscript preparation, or the decision to submit the manuscript for publication.

\section{References}

1. National Center for Health Statistics (US). Health, United States, 2015: With Special Feature on Racial and Ethnic Health Disparities. Hyattsville, MD: National Center for Health Statistics (US); 2016. http://www.ncbi.nlm.nih.gov/books/ NBK367640/. Accessed November 2, 2016.

2. Weiss AJ, Elixhauser A. Overview of Hospital Stays in the United States, 2012: Statistical Brief \#180. In: Healthcare Cost and Utilization Project (HCUP) Statistical Briefs. Rockville (MD): Agency for Healthcare Research and Quality (US); 2006. http:// www.ncbi.nlm.nih.gov/books/NBK259100/. Accessed November 2, 2016.

3. Jencks SF, Cuerdon T, Burwen DR, et al. Quality of medical care delivered to medicare beneficiaries: A profile at state and national levels. JAMA. 2000;284(13):1670-1676.

4. Covinsky KE, Pierluissi E, Johnston C. Hospitalization-associated disability: "She was probably able to ambulate, but I'm not sure." JAMA. 2011;306(16):1782-1793.

5. Creditor MC. Hazards of Hospitalization of the Elderly. Ann Intern Med 1993;118(3):219-223.

6. Graf C. Functional decline in hospitalized older adults. Am J Nurs. 2006;106(1) 58-67, NaN-68

7. Wachter RM, Goldman L. The emerging role of "hospitalists" in the American health care system. N Engl J Med. 1996;335(7):514-517.

8. Lindenauer PK, Pantilat SZ, Katz PP, Wachter RM. Hospitalists and the practice of inpatient medicine: results of a survey of the National Association of Inpatient Physicians. Ann Intern Med. 1999;130(4 Pt 2):343-349.

9. Wachter RM. The hospitalist movement 5 years later. JAMA. 2002;287(4):487.

10. Shank B. 2016: Celebrating 20 years of hospital medicine and looking toward a bright future. Hosp Natl Assoc Inpatient Physicians. 2016. http://www.the-hospitalist.org/hospitalist/article/121925/2016-celebrating-20-years-hospital-medicine-and-looking-toward-bright. Accessed June 2, 2017.

11. Retooling for an Aging America: Building the Health Care Workforce. Washington, DC.: National Academies Press; 2008. http://www.nap.edu/catalog/12089. Ac cessed November 2, 2016.

12. Boult C, Counsell SR, Leipzig RM, Berenson RA. The urgency of preparing primary care physicians to care for older people with chronic illnesses. Health Aff Proj Hope. 2010;29(5):811-818.

13. Warshaw GA, Bragg EJ, Thomas DC, Ho ML, Brewer DE, Association of Direc tors of Geriatric Academic Programs. Are internal medicine residency programs adequately preparing physicians to care for the baby boomers? A national survey from the Association of Directors of Geriatric Academic Programs Status of Geriatrics Workforce Study. J Am Geriatr Soc. 2006;54(10):1603-1609.

14. Tanner CE, Eckstrom E, Desai SS, Joseph CL, Ririe MR, Bowen JL. Uncovering frustrations: A qualitative needs assessment of academic general internists as geriatric care providers and teachers. J Gen Intern Med. 2006;21(1):51-55.

15. Warshaw GA, Bragg EJ, Brewer DE, Meganathan K, Ho M. The development of academic geriatric medicine: progress toward preparing the nation's physicians to care for an aging population. J Am Geriatr Soc. 2007;55(12):2075-2082.

16. Fox MT, Sidani S, Persaud M, et al. Acute care for elders components of acute geriatric unit care: Systematic descriptive review. J Am Geriatr Soc. 2013;61(6):939-946.

17. Palmer RM, Landefeld CS, Kresevic D, Kowal J. A medical unit for the acute care of the elderly. J Am Geriatr Soc. 1994:42(5):545-552.

18. Hung WW, Ross JS, Farber J, Siu AL. Evaluation of the Mobile Acute Care of the Elderly (MACE) service. JAMA Intern Med. 2013;173(11):990-996.

19. Sennour Y, Counsell SR, Jones J, Weiner M. Development and implementation of a proactive geriatrics consultation model in collaboration with hospitalists. J Am Geriatr Soc. 2009;57(11):2139-2145.

20. Ellis G, Whitehead MA, O'Neill D, Langhorne P, Robinson D. Comprehensive geriatric assessment for older adults admitted to hospital. Cochrane Database Syst Rev. 2011;(7):CD006211.

21. Mattison MLP, Catic A, Davis RB, et al. A standardized, bundled approach to providing geriatric-focused acute care. J Am Geriatr Soc. 2014;62(5):936-942. doi:10.1111/jgs.12780

22. Wenger NS, Shekelle PG. Assessing care of vulnerable elders: ACOVE project overview. Ann Intern Med. 2001;135(8 Pt 2):642-646.

23. Wenger NS, Roth CP, Shekelle P, ACOVE Investigators. Introduction to the assessing care of vulnerable elders-3 quality indicator measurement set. J Am Geriatr Soc. 2007;55 Suppl 2:S247-S252.

24. Reuben DB, Roth C, Kamberg C, Wenger NS. Restructuring primary care practic es to manage geriatric syndromes: the ACOVE-2 intervention. J Am Geriatr Soc. 2003;51(12):1787-1793
25. Askari M, Wierenga PC, Eslami S, Medlock S, De Rooij SE, Abu-Hanna A. Studies pertaining to the ACOVE quality criteria: a systematic review. Int J Qual Health Care. 2012;24(1):80-87.

26. Arora VM, McGory ML, Fung CH. Quality indicators for hospitalization and surgery in vulnerable elders. J Am Geriatr Soc. 2007;55 Suppl 2:S347-S358.

27. Arora VM, Johnson M, Olson J, et al. Using assessing care of vulnerable elders quality indicators to measure quality of hospital care for vulnerable elders. J Am Geriatr Soc. 2007;55(11):1705-1711.

28. Sands M, Dantoc B, Hartshorn A, Ryan C, Lujic S. Single Question in Delirium (SQiD): testing its efficacy against psychiatrist interview, the Confusion Assessment Method and the Memorial Delirium Assessment Scale. Palliat Med. 2010;24(6):561-565.

29. Inouye SK, van Dyck CH, Alessi CA, Balkin S, Siegal AP, Horwitz RI. Clarifying confusion: the confusion assessment method. A new method for detection of delirium. Ann Intern Med. 1990;113(12):941-948.

30. Puelle MR, Kosar CM, Xu G, et al. The language of delirium: Keywords for identifying delirium from medical records. J Gerontol Nurs. 2015;41(8):34-42.

31. Quan H, Sundararajan V, Halfon P, et al. Coding algorithms for defining comorbidities in ICD-9-CM and ICD-10 administrative data. Med Care. 2005;43(11): 1130-1139

32. Boult C, Boult L, Morishita L, Smith SL, Kane RL. Outpatient geriatric evaluation and management. J Am Geriatr Soc. 1998;46(3):296-302.

33. Wenger NS, Roth CP, Shekelle PG, et al. A practice-based intervention to improve primary care for falls, urinary incontinence, and dementia. J Am Geriatr Soc. 2009;57(3):547-555.

34. Geerts WH. Prevention of Venous Thromboembolism: American College of Chest Physicians Evidence-Based Clinical Practice Guidelines (8th Edition). Chest J. 2008;133(6_suppl):381S.

35. Rosenman M, Liu X, Phatak H, et al. Pharmacological prophylaxis for venous thromboembolism among hospitalized patients with acute medical illness: An electronic medical records study. Am J Ther. 2016;23(2):e328-e335

36. Ghanem A, Artime C, Moser M, Caceres L, Basconcillo A. Holy moley! Take out that foley! Measuring compliance with a nurse driven protocol for foley catheter removal to decrease utilization. Am J Infect Control. 2015;43(6):S51.

37. Cornia PB, Amory JK, Fraser S, Saint S, Lipsky BA. Computer-based order entry decreases duration of indwelling urinary catheterization in hospitalized patients. Am J Med. 2003;114(5):404-407.

38. Huang W-C, Wann S-R, Lin S-L, et al. Catheter-associated urinary tract infections in intensive care units can be reduced by prompting physicians to remove unnecessary catheters. Infect Control Hosp Epidemiol. 2004:25(11):974-978.

39. Topal J, Conklin S, Camp K, Morris V, Balcezak T, Herbert P. Prevention of nosocomial catheter-associated urinary tract infections through computerized feedback to physicians and a nurse-directed protocol. Am J Med Qual. 2005;20(3): 121-126.

40. Zisberg A, Shadmi E, Gur-Yaish N, Tonkikh O, Sinoff G. Hospital-associated functional decline: the role of hospitalization processes beyond individual risk factors. J Am Geriatr Soc. 2015;63(1):55-62.

41. Inouye SK, Bogardus ST, Baker DI, Leo-Summers L, Cooney LM. The Hospital Elder Life Program: a model of care to prevent cognitive and functional decline in older hospitalized patients. Hospital Elder Life Program. J Am Geriatr Soc. 2000;48(12):1697-1706.

42. Hoyer EH, Friedman M, Lavezza A, et al. Promoting mobility and reducing length of stay in hospitalized general medicine patients: A quality-improvement project. J Hosp Med. 2016;11(5):341-347.

43. Mahoney FI, Barthel DW. Functional evaluation: the barthel index. Md State Med J. 1965;14:61-65.

44. Katz S, Ford AB, Moskowitz RW, Jackson BA, Jaffe MW. Studies of illness in the aged. the index of adl: a standardized measure of biological and psychosocial function. JAMA. 1963;185:914-919.

45. Tinetti ME. Performance-oriented assessment of mobility problems in elderly patients. J Am Geriatr Soc. 1986;34(2):119-126.

46. Smith R. Validation and Reliability of the Elderly Mobility Scale. Physiotherapy. 1994;80(11):744-747.

47. Inouye SK, Foreman MD, Mion LC, Katz KH, Cooney LM. Nurses' recognition of delirium and its symptoms: comparison of nurse and researcher ratings. Arch Intern Med. 2001;161(20):2467-2473.

48. Gustafson Y, Brännström B, Norberg A, Bucht G, Winblad B. Underdiagnosis and poor documentation of acute confusional states in elderly hip fracture patients. $J$ Am Geriatr Soc. 1991;39(8):760-765

49. Brenner SK, Kaushal R, Grinspan Z, et al. Effects of health information technology on patient outcomes: a systematic review. J Am Med Inform Assoc. 2016;23(5):1016-1036 